\title{
Importance of Effective Teaching in Robot Motor Skill Learning
}

\author{
Joe Cloud \\ Computer Science \& Engineering \\ University of Texas at Arlington \\ Arlington, TX, USA \\ joe.cloud@mavs.uta.edu \\ James Brady \\ Computer Science \& Engineering \\ University of Texas at Arlington \\ Arlington, TX, USA \\ james.brady2@mavs.uta.edu
}

\author{
Michail Theofanidis \\ Computer Science \& Engineering \\ University of Texas at Arlington \\ Arlington, TX, USA \\ michail.theofanidis@mavs.uta.edu \\ Fillia Makedon \\ Computer Science \& Engineering \\ University of Texas at Arlington \\ Arlington, TX, USA \\ makedon@uta.edu
}

\begin{abstract}
It is generally accepted in the field of human-robot interaction (HRI) that the role of a human teacher is critical for robot environmental adaptation. As such, the ability of a robot to adapt in a novel environment is based on the capabilities of the human teacher. In this paper, we propose a metric for robots to evaluate the quality of a human teacher. We illustrate a scenario where the Sawyer Robotic Arm must learn to grasp a cup according to the demonstration of a good and a bad human teacher.
\end{abstract}

\section{CCS CONCEPTS}

- Computer systems organization $\rightarrow$ Robotics; Robotic autonomy; • Computing methodologies $\rightarrow$ Reinforcement learning; Learning from demonstrations.

\section{KEYWORDS}

human-robot interaction, kinesthetic teaching, machine learning, dynamic movement primitives, learning from demonstration

ACM Reference Format:

Joe Cloud, Michail Theofanidis, James Brady, and Fillia Makedon. 2019. Importance of Effective Teaching in Robot Motor Skill Learning. In The 12th PErvasive Technologies Related to Assistive Environments Conference (PETRA '19), June 5-7, 2019, Rhodes, Greece. ACM, New York, NY, USA, 4 pages. https://doi.org/10.1145/3316782.3322775

\section{INTRODUCTION}

In most industrial facilities, robots need to be programmed by skilled human programmers, who have the capacity to decompose and describe complicated motions. In recent years, there is a trend of research to enhance the motor skill learning capabilities of robots through the use of Learning from Demonstration (LfD) approaches [2], [4], [16]. Recent LfD methods rely on supervised learning [2],

Permission to make digital or hard copies of all or part of this work for personal or classroom use is granted without fee provided that copies are not made or distributed for profit or commercial advantage and that copies bear this notice and the full citation on the first page. Copyrights for components of this work owned by others than the author(s) must be honored. Abstracting with credit is permitted. To copy otherwise, or republish, to post on servers or to redistribute to lists, requires prior specific permission and/or a fee. Request permissions from permissions@acm.org.

PETRA '19, fune 5-7, 2019, Rhodes, Greece

(C) 2019 Copyright held by the owner/author(s). Publication rights licensed to ACM. ACM ISBN 978-1-4503-6232-0/19/06 . \$ \$15.00

https://doi.org/10.1145/3316782.3322775
[17] or goal oriented approaches [5], [6] and learn information either in Cartesian space [11] or joint space [12] through demonstrations provided by a human teacher. The quality of these demonstrations is important, as low quality demonstrations can lead to poor learner performance [1]. The combination of goal oriented machine learning algorithms, such as Reinforcement Learning (RL) [15] and the Dynamic Movement Primitive (DMP) framework has shown many promising results because it has enabled robots to learn complicated motor skills [9], [10], in addition to allowing a learner to improve beyond the original demonstration which is critical for overcoming low quality demonstrations.

The fusion of the DMP framework with RL algorithms, such as PoWER [13], has created a unique new paradigm. Initially the robotic system performs imitation learning by using the Locally Weighted Regression algorithm (LWR) [14] to produce a generalized state-action mapping policy. This initial policy is later adapted with RL, so that the robot can respond to different environmental stimuli [3]. To further elaborate, a human initially provides demonstration data which is used to compute an initial policy. This policy is later converted to the optimal policy with RL [5], [8]. Thus, given that there is one and only one optimal policy per environmental stimuli, the amount of episodes which are required to convert a policy to the optimal one depends on the quality of the demonstration. In this paper we propose a method to quantify the quality of demonstrations based on the number of RL episodes which are necessary to convert a suboptimal policy to the optimal one.

\section{RELATED WORK}

In this section we will briefly describe the DMP framework to learn motor policies and the PoWER algorithm to adapt policies to the optimal ones. We utilize these methods to perform the experiments in the next sections of the paper.

\subsection{Dynamic Motion Primitives Formalization}

Dynamic Movement Primitives (DMPs) are a set of differential equations based on the spring-damper system and are used to generate discrete [and rhythmic] movements [3]. The goal in using DMPs is to find the forcing term $f$ in equation 1 with which we can generate the desired motions. In this paper, we focus only on attractor systems. 


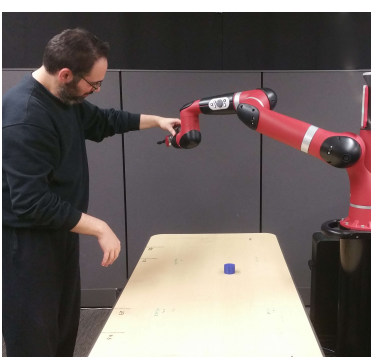

(a) Bad teacher

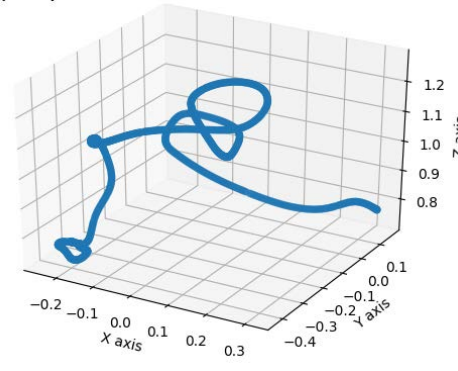

(b) Bad teacher trajectory

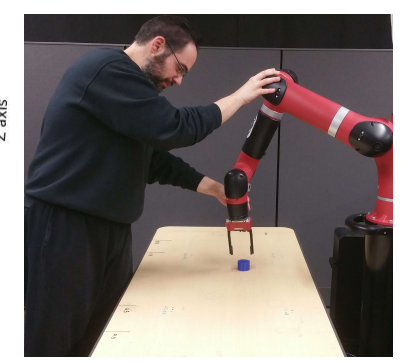

(c) Good teacher

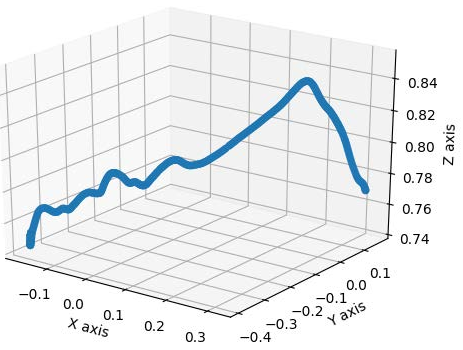

(d) Good teacher trajectory

Figure 1: Teacher demonstrating bad and good trajectories to the robot

$$
\begin{gathered}
\tau \dot{u}=a_{z}\left(\beta_{z}(g-x)-u\right)+\left(g-x_{0}\right) f \\
\tau \dot{x}=u
\end{gathered}
$$

In equations 1 and 2, $x$ and $u$ are the position and velocity of the robot's joints. $\tau$ is the duration of the demonstration, $x_{0}$ and $g$ are the initial and target positions of the robot's joint. The terms $a_{z}$ and $\beta_{z}$ are control gains selected to make the system critically damped and $f$ acts as the control input that drives the system towards the goal. The forcing term is further defined as:

$$
f(s)=\frac{\sum_{i} w_{i} \psi_{i}(s) s}{\sum_{i} \psi_{i}(s)}
$$

Where $\psi_{i}(s)=\exp \left(-h_{i}\left(s-c_{i}\right)^{2}\right)$ are Gaussian basis functions, with width $h_{i}$ and centers $c_{i}$, while $w_{i}$ are adjustable weights that changes the shape of the Gaussians over the $s$ domain. Note that the forcing terms is dependent on $s$, which is derived from the equation:

$$
\tau \dot{s}=\alpha s
$$

In equation $4, s$ represents a variable that starts from time $t=0$ and becomes zero at $t=\tau$. To learn the motor policy from a demonstration, we record $x(t), u(t)$ and $\dot{u}(t)$ for the duration of the trajectory. From this, we compute $s$ according to equation 4 by setting $\alpha$ such that $s$ becomes zero at final time. Note that since the system learns motor policies in joint space, equation 4 behaves the same for all joints, while equations 1, 2, and 3 behave independently for each joint. To continue with the computation of the motor policy, we then estimate $f$ from equation 1 and we learn its Gaussian representation by performing regression using the Locally Weighted Regression algorithm (LWR) as suggested in [3].

\subsection{Adapting Learned Policies with Reinforcement Learning}

To adapt motor policies learned from DMPs we utilize the PoWER algorithm as described in [7]. A pseudo-code of the implemented RL algorithm can be seen in the section Algorithm 1. The general goal of the algorithm is to find a trajectory that maximizes the reward. Different trajectories can be generated by using equation 1 . Note that we consider $w_{i}$ as the open parameter of the policy for policy search.

\begin{abstract}
Initialization: Policy with parameters $\theta_{0}$;
while $\theta_{k}$ has not converged do

Create sample rollouts using:

$\alpha=\left(\theta_{k}+\epsilon_{t}\right) \psi_{i}(s), \epsilon \sim N\left(0, \sigma^{2}\right)$

Estimate the importance of each rollout:

$Q(s, \alpha, t)=\sum_{t=0}^{T} r\left(s_{t}, \alpha_{t}, t\right)$

Discard the rollouts with low reward.

Update using the following rule:

$\theta_{k+1}=\theta_{k}+\frac{\sum_{t=0}^{T} \epsilon_{t} Q(s, \alpha, t)}{\sum_{t=0}^{T} Q(s, \alpha, t)}$

end

Algorithm 1: Policy learning by weighted exploration.

The initial sub-optimal policy $\hat{\pi}_{t}(s, \alpha)$ can be expressed according to equation 3 as a battery of Gaussians that expand in the $s$ domain. As such, we utilize the PoWER algorithm to find the optimal set of parameters $w_{i}$ that generate the optimal policy $\pi_{t}^{*}(s, \alpha)$ which in turn drives the robot to the new goal $\hat{\delta}$. Note, that if the learned DMP can generalize its motor policy to reach the goal, the RL is not expected to drastically modify the Gaussians of the policy. On the other hand, if the initial policy $\hat{\pi}_{t}(s, \alpha)$ can not generalize its action space to reach the goal, the RL module must alter the weights of the Gaussians accordingly through a number of episodes.
\end{abstract}

\section{EXPERIMENTAL RESULTS}

In this section we discuss the setup and describe the results of our experiments. The analysis of our results depends on a hypothesis presented in the following subsection.

\subsection{Hypothesis}

Our hypothesis is that an encapsulated demonstration conducted by a good teacher will require fewer RL episodes then that of a bad teacher. The proposed reasoning for this is that a bad demonstration's encapsulated DMP will initialize to a less optimal policy then that of a good DMP due to the ambiguity and unnecessary complexity presented in a poor demonstration [1]. In order to test this, we expect that a good initial policy will require fewer episodes of RL to converge to an optimal solution as a poor initial policy will need more exploration, and thus, more episodes to reach the stopping criteria. 


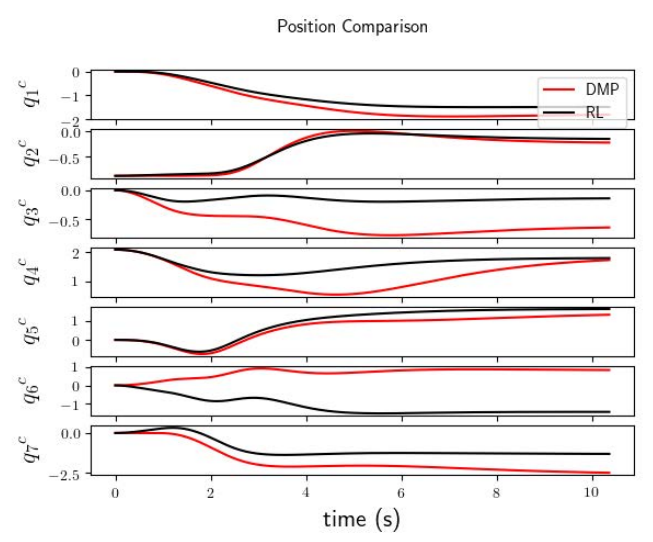

(a) Bad teacher trajectory

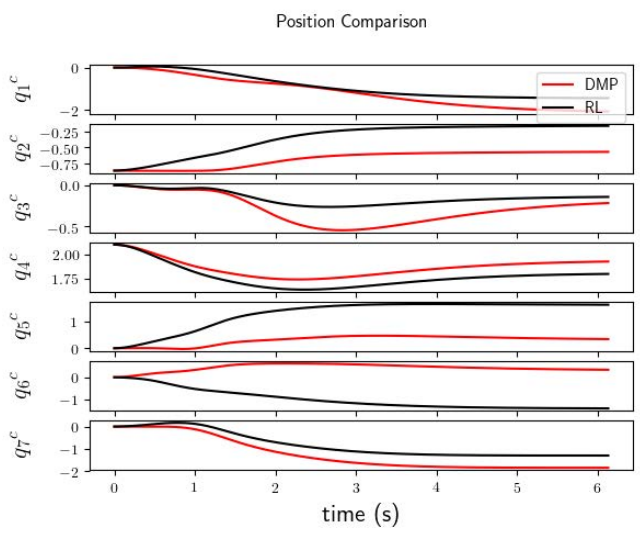

(b) Good teacher trajectory

Figure 2: Trajectories at each stage of optimization in joint-space

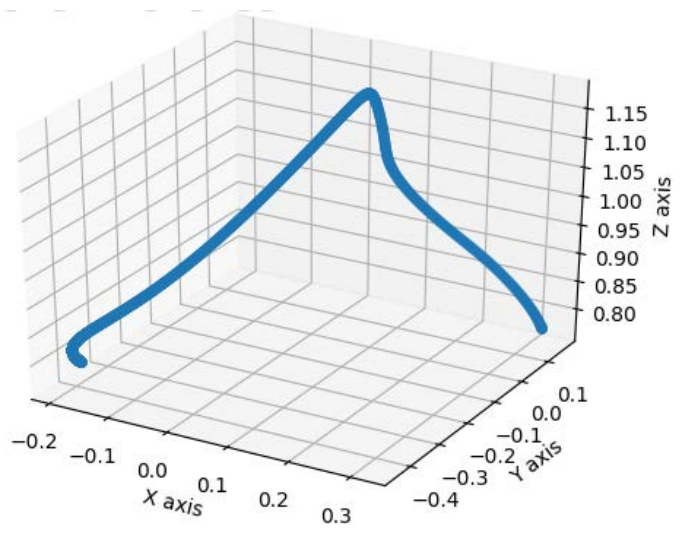

(a) Bad teacher trajectory

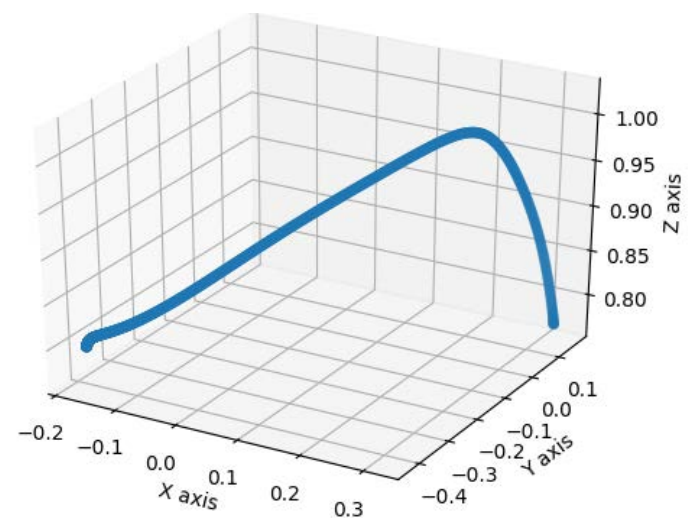

(b) Good teacher trajectory

Figure 3: Cartesian representation of the trajectories post-RL

\subsection{Experimental Setup}

A human teacher initially performs one demonstration for two different scenarios. In the first scenario, the teacher moves the robotic arm in a direct and efficient manner. This is considered the good trajectory. In the next scenario, the teacher performs convoluted movements to intentionally make it difficult for the arm to efficiently generalize the primitive, this is shown in figure 1 . With these demonstrations recorded for the two different scenarios, the trajectories can be encapsulated by respective DMPs to compute the initial policy.

\subsection{Experimental Results}

Figure 1 visualizes the demonstrated trajectories in Cartesian space for both the bad and good teacher. Clearly, the bad teacher trajectory is of a convoluted form as opposed to that of the good teacher, though they both eventually reach the same goal location.

In figure 2, we present a comparison between the initial suboptimal policy, denoted as DMP in red, and the optimized policy denoted as RL in black. For many of the joints, the joint-space trajectories diverge as an optimal policy is discovered. This is clearly seen for both the good and bad teacher, though when the forward kinematics is applied to visualize the trajectory in Cartesian space, we see both good and bad initial policies have reached an optimal state as defined by our stopping criteria. The bad teacher trajectory has clearly been smoothed heavily. The smoothing can be reduced with the introduction of additional Gaussians, though learning will take more time.

Though they both reach optimal states, the question of our hypothesis is whether or not the effectiveness of a demonstration impacts the efficiency during optimization. In table 1 , we find that a bad policy requires many more episodes to converge to a solution than that of a good initial policy. As shown in table 1 , Joint 1 is the only joint where the initial policy of the bad demonstration outperforms that of the good demonstration, all other joints in the good demonstration strongly outperformed or tied with the joint episodes of the bad demonstration. 
Table 1: Episodes for each joint for each demonstration

\begin{tabular}{|l|r|r|r|r|r|r|r|r|}
\hline Joint & $\mathbf{1}$ & $\mathbf{2}$ & $\mathbf{3}$ & $\mathbf{4}$ & $\mathbf{5}$ & $\mathbf{6}$ & $\mathbf{7}$ & Total \\
\hline Bad & 3 & 1 & 2 & 24 & 7 & 5 & 10 & 52 \\
\hline Good & 18 & 1 & 8 & 1 & 2 & 3 & 1 & 34 \\
\hline
\end{tabular}

\section{CONCLUSION AND FUTURE WORK}

Traditionally, research on the area of HRI focuses on robot adaptation, generalization, and performance. However, most HRI research is human-centric and not robot-centric, as we perceive robot adaptation to be an inherent characteristic that originates from the capabilities of the robot and not of the environment. It is important to recognize the impact of ineffective demonstrations on a robot's ability to encapsulate and learn better policies. By doing so, this paper highlights the need for concrete metrics to further quantify the quality of raw demonstrations. In this paper, we proposed an approach to assess the quality of teacher demonstrations in order to highlight that robot environmental adaptation can be improved by changing the parameters that exist in the environment and are not part of the robot. To improve upon this work, we will explore the development of more principled methods for evaluating the quality of demonstrations. Doing so allows us to explore situations where a robot may need to repair skills and acquire additional information about the task in cases of ambiguity.

\section{ACKNOWLEDGMENTS}

This work is supported in part by the National Science Foundation under award numbers 1338118 and 1719031. Any opinions, findings, and conclusions or recommendations expressed in this publication are those of the author(s) and do not necessarily reflect the views of the National Science Foundation.

\section{REFERENCES}

[1] B. D. Argall, S. Chernova, M. Veloso, and B. Browning. A survey of robot learning from demonstration. Robotics and autonomous systems, 57(5):469-483, 2009

[2] S. Calinon, F. Guenter, and A. Billard. On learning, representing, and generalizing a task in a humanoid robot. IEEE Transactions on Systems, Man, and Cybernetics, Part B (Cybernetics), 37(2):286-298, 2007.

[3] A. J. Ijspeert, J. Nakanishi, H. Hoffmann, P. Pastor, and S. Schaal. Dynamical movement primitives: learning attractor models for motor behaviors. Neural computation, 25(2):328-373, 2013.

[4] L. Jin, S. Li, J. Yu, and J. He. Robot manipulator control using neural networks: A survey. Neurocomputing, 285:23-34, 2018.

[5] J. Kober, J. A. Bagnell, and J. Peters. Reinforcement learning in robotics: A survey The International fournal of Robotics Research, 32(11):1238-1274, 2013.

[6] J. Kober and J. Peters. Learning motor primitives for robotics. In Robotics and Automation, 2009. ICRA'09. IEEE International Conference on, pages 2112-2118. IEEE, 2009.

[7] J. Kober and J. Peters. Imitation and reinforcement learning. IEEE Robotics \& Automation Magazine, 17(2):55-62, 2010.

[8] J. Kober, A. Wilhelm, E. Oztop, and J. Peters. Reinforcement learning to adjust parametrized motor primitives to new situations. Autonomous Robots, 33(4):361$379,2012$.

[9] K. Muelling, J. Kober, and J. Peters. Learning table tennis with a mixture of motor primitives. In Humanoid Robots (Humanoids), 2010 10th IEEE-RAS International Conference on, pages 411-416. IEEE, 2010.

[10] K. Mülling, J. Kober, O. Kroemer, and J. Peters. Learning to select and generalize striking movements in robot table tennis. The International fournal of Robotics Research, 32(3):263-279, 2013.

[11] P. Pastor, H. Hoffmann, T. Asfour, and S. Schaal. Learning and generalization of motor skills by learning from demonstration. In Robotics and Automation, 2009. ICRA'09. IEEE International Conference on, pages 763-768. IEEE, 2009.

[12] P. Pastor, M. Kalakrishnan, S. Chitta, E. Theodorou, and S. Schaal. Skill learning and task outcome prediction for manipulation. In Robotics and Automation (ICRA),
2011 IEEE International Conference on, pages 3828-3834. IEEE, 2011.

[13] S. Schaal. Is imitation learning the route to humanoid robots? Trends in cognitive sciences, 3(6):233-242, 1999.

[14] S. Schaal, C. G. Atkeson, and S. Vijayakumar. Scalable techniques from nonparametric statistics for real time robot learning. Applied Intelligence, 17(1):49-60, 2002.

[15] R. S. Sutton and A. G. Barto. Introduction to Reinforcement Learning. MIT Press, Cambridge, MA, USA, 1st edition, 1998

[16] M. Theofanidis, J. Cloud, A. R. Babu, J. Brady, and F. Makedon. A human robot interaction framework for robotic motor skill learning. In Proceedings of the 11th PErvasive Technologies Related to Assistive Environments Conference, PETRA '18, pages 110-111, New York, NY, USA, 2018. ACM.

[17] M. Theofanidis, S. I. Sayed, J. Cloud, J. Brady, and F. Makedon. Kinematic estimation with neural networks for robotic manipulators. In International Conference on Artificial Neural Networks, pages 795-802. Springer, 2018. 